\title{
The effect of consumption volume on profile and liking of oral nutritional supplements of varied sweetness: sequential profiling and boredom tests
}

Article

Accepted Version

Methven, L., Rahelu, K., Economoua, N., Kinneavya, L., Ladbrooke-Davis, L., Kennedy, O., Mottram, D. S. and Gosney, M. (2010) The effect of consumption volume on profile and liking of oral nutritional supplements of varied sweetness: sequential profiling and boredom tests. Food Quality and Preference, 21 (8). pp. 948-955. ISSN 0950-3293 doi: https://doi.org/10.1016/j.foodqual.2010.04.009 Available at https://centaur.reading.ac.uk/8137/

It is advisable to refer to the publisher's version if you intend to cite from the work. See Guidance on citing.

To link to this article DOI: http://dx.doi.org/10.1016/j.foodqual.2010.04.009

Publisher: Elsevier

All outputs in CentAUR are protected by Intellectual Property Rights law, including copyright law. Copyright and IPR is retained by the creators or other copyright holders. Terms and conditions for use of this material are defined in the End User Agreement. 


\section{www.reading.ac.uk/centaur}

\section{CentAUR}

Central Archive at the University of Reading

Reading's research outputs online 
1 The Effect of Consumption Volume on Profile and Liking of Oral Nutritional

2 Supplements of Varied Sweetness: Sequential Profiling and Boredom Tests 3

L. Methven ${ }^{a^{\star}}$, K. Rahelua , N. Economou ${ }^{a}$, L. Kinneavy ${ }^{a}$, L. Ladbrooke-Davis ${ }^{a}$, O. B. Kennedy $^{a}$, D. S. Mottram ${ }^{\text {a }}$, M. A. Gosney

${ }^{a}$ Department of Food and Nutritional Sciences, University of Reading, Whiteknights,

8 Reading RG6 6AP, UK

$9 \quad{ }^{b}$ Clinical Health Sciences, University of Reading, London Road, Reading, Berkshire, RG1

$105 \mathrm{AQ}$

$11{ }^{*}$ Corresponding author. Fax: $+44(0) 118$ 9310080. E-mail address:

12 I.methven@reading.ac.uk

13 Running Title: Sequential Profile and Liking of Oral Nutritional Supplements

Abstract

Oral nutrition supplements (ONS) are routinely prescribed to those with, or at risk of,

17 malnutrition. Previous research identified poor compliance due to taste and sweetness.

18 This paper investigates taste and hedonic liking of ONS, of varying sweetness and metallic

19 levels, over consumption volume; an important consideration as patients are prescribed

20 large volumes of ONS daily. A sequential descriptive profile was developed to determine

21 the perception of sensory attributes over repeat consumption of ONS. Changes in liking of

22 ONS following repeat consumption were characterised by a boredom test. Certain flavour

23 (metallic taste, soya milk flavour) and mouthfeel (mouthdrying, mouthcoating) attributes

24 built up over increased consumption volume $(p \leq 0.002)$. Hedonic liking data from two

25 cohorts, healthy older volunteers $(n=32$, median age 73$)$ and patients $(n=28$, median age

26 85), suggested such build-up was disliked. Efforts made to improve the palatability of ONS 
27 must take account of the build up of taste and mouthfeel characteristics over increased

28 consumption volume.

Keywords: oral nutrition supplements, sensory attributes, sequential profile, boredom test

\section{Introduction}

Malnutrition is a recognised problem in the elderly population, especially in hospitalised subjects; $60 \%$ of older people are at risk of malnutrition, or their situation worsening, in hospital (Age Concern, 2006). Identification and treatment of malnutrition in this high-risk group is extremely important to reduce the risk of disease, prevent worsening of any existing conditions and to maintain an optimum quality of life (O'Flynn, Peake, Hickson, Foster and Frost, 2005). Oral nutrition supplements (ONS) are supplementary oral dietary "food" routinely prescribed in-between meals to help improve the nutritional status of those with, or at risk of, malnutrition (Lochs et al, 2006). A review of sixty-two intervention trials (10,187 participants) by Milner, Potter, Vivanti and Avenell (2002) found ONS supplementation to produce consistent weight gain (in 42 trials), and decreased relative risk for mortality in trials where participants were undernourished $(n=2461)$.

44 It has been suggested that the success of ONS may depend on consumption of sufficient quantities over an extended time period (Rahemtulla et al., 2005). A study investigating the effect of quantity of ONS consumed on weight loss and lean tissue in cancer patients, identified a failure to achieve the desired supplement intake prevented patients from obtaining important clinical benefits (Fearon et al., 2003). Gosney (2003) indicated that

49 compliance of ONS can be low, thus limiting the success that can be achieved from prescribing ONS. A 24-hour study of 96 elder care ward patients found that two-thirds of

51 the patients given ONS drank less than $50 \%$ of the carton resulting in $63 \%$ of ONS being wasted. Poor compliance with ONS has been demonstrated previously; Nolan (1999) 
53 reported average wastage of two different ONS to be $41 \%$ and $44 \%$ and Stableforth

54 (1986) showed that elderly patients with femoral neck fractures only tolerated limited

55 amounts of ONS which meant that large calorie deficits remained. Bolton et al (1992)

56 compared the long term palatability of three commercial ONS products with cancer

57 patients and found that $54 \%$ of patients discontinued the trial for flavour reasons. In the

582003 study, Gosney (2003) found the greatest wastage of ONS was found in patients who

59 disliked the taste (72\%). Of the $67 \%$ of patients who completed questionnaires, $56 \%$ said

60 they did not like the products and specific dislikes were taste (25\%), texture (19\%) and

61 sweetness (38\%). Other factors that were thought to decrease compliance with ONS

62 include a lack of thirst, chemosensory changes associated with ageing, the unfamiliarity of

63 cartons to elderly people, in comparison to the frequently available cups of tea, and

64 frequent spillage from cartons as a result of decreased dexterity (Gosney, 2003). Taste

65 fatigue, which tends to occur when ONS are consumed regularly over prolonged periods,

66 is thought to contribute to poor compliance (Rahemtulla et al., 2005).

67 A recent study reported age-related differences in preferred sweetness level, which were 68 in-line with increased detection and recognition thresholds for sweetness; an overall dislike

69 of ONS and dislike of the sweetness level of ONS vanilla products (Law, Gosney and 70 Kennedy, 2006; Law 2006). Literature on age related taste threshold changes, and 71 potential affects on food preference are somewhat contradictory. A number of studies have 72 shown sweet taste threshold to increase with age (Zandstra and de Graaf, 1998; Mojet, 73 Heidema and Christ-Hazelhof, 2003; Fukunaga, Uematsu and Sugimoto, 2005), whilst 74 other studies have found no significant age-related decline in sweet perception (Kaneda et 75 al, 2000; Koskinen, Kälviänen and Tuorila 2003). Mojet, Christ-Hazelhof and Heidema 76 (2005) found no correlation between threshold sensitivity and optimal liking concentration for any basic taste stimuli; however Zandstra and de Graaf (1998) did find a trend for high 
78 optimal concentrations of sucrose and orange flavour in drinks for elderly subjects

79 compared to younger adults.

80 Development of ONS with lower sweetness, by replacing sucrose with an alternative 81 saccharide, palatinose ${ }^{\mathrm{TM}}$ ( $\alpha$-D-glucopyranosyl-1,6-fructose), led to segmentation in

82 preference between consumers who liked the less sweet variants, and those who liked the 83 sweeter control (Methven et al, 2008). The study noted that further work was needed to investigate if there was a difference in liking between ONS of different sweetness levels on

consumption of greater quantities, in line with the typical pack size $(200 \mathrm{ml})$. However, there appears to be no study in the literature which examines the specific sensory attributes of ONS or their affect on liking over increasing consumption volume; this latter point is likely to be extremely important in identifying potential reasons for the rejection of ONS, which may arise when greater quantities of ONS are consumed.

In order to measure change in sensory perception over consumption time, time intensity profiling (TI) is typically used (Duizer, Bloom and Findlay, 1997), however TI can only characterise a maximum of two attributes per sample. A temporal dominance method (Labbe, Schlich, Pineau, Gilbert and Martin, 2009) has been developed recently, although one potential drawback of this method for products such as ONS could be that attributes of secondary rather than primary dominance might be important determinants of product liking. A previous study used progressive profiling (Jack, Piggott and Paterson, 1994) to profile the textural attributes of hard cheese during mastication. In the present study this idea has been progressed, with the help of Compusense, to a sequential profiling method where up to five attributes are scored over consecutive tastings, at regimented time intervals.

The present study aimed to investigate the effect of consumption volume on the sensory profile and liking of ONS. In addition the study aimed to investigate if modifications of sweetness and metallic levels could improve the hedonic liking of ONS. 


\section{Materials and methods}

105 The commercial ONS (CONS) used was Ensure Vanilla Plus (Abbott Nutrition, 106 Maidenhead, UK), and Lactisole (sodium 2-(4-methoxyphenoxy)-propanoate) was used as 107 a sweetness suppressor (Domino Sugar, American Sugar Refining, USA). Standard 108 ingredients used in the manufacture of ONS were as follows : glucose syrup (Cerestar 109 01921, Cargill, Manchester, UK), sucrose (Tate and Lyle, London, UK), high oleic 110 sunflower oil, canola oil, and rape seed oil (Cargill, Liverpool, UK), sodium caseinate 111 (Bacarel, Stone, UK), milk protein concentrate (MPC85, Bacarel, Stone, UK), soy protein 112 isolate (ProFit SI90, Food Ingredient Technologies, Bedfordshire, UK); soy lecithin 113 (Emulpur IP, Cargill, Hamburg, Germany); commercial blends of emulsifier, vanilla flavour, 114 vitamins and minerals were supplied by Abbott (Abbott Nutrition, Columbus, USA). 115 Mineral water (Harrogate Spa, UK) and medium sliced white bread (Hovis, Windsor, UK) were used as palate cleansers in sensory testing. Sucrose (Tate and Lyle, London, UK)

117 and iron sulphate heptahydrate (Fluka, Sigma Aldrich, Germany) were used for taste 118 threshold tests.

\subsection{Manufacture of ONS modifications}

120 Preparation of suppressed sweetness ONS (SSONS) was carried out by adding the 121 sweetness suppressor lactisole to the commercial vanilla ONS products $(0.003 \mathrm{mg}$ 122 lactisole/100ml Ensure Plus Vanilla). In addition, ONS samples were manufactured on a 123 pilot scale ultra heat treatment (UHT) plant. The standard formulation (PPSONS) 124 consisted, per 100g, of glucose syrup (17g), sodium caseinate (3.5 g), sucrose (2 g), oil 125 blend $(4.4 \mathrm{~g})$, milk protein concentrate $(1.8 \mathrm{~g})$, soy protein isolate $(1.3 \mathrm{~g})$ and a commercial 126 blend of emulsifier, flavour, vitamins and minerals. Ingredients were blended at $60{ }^{\circ} \mathrm{C}$ prior 127 to ultra heat treatment by indirect steam injection at $140^{\circ} \mathrm{C}$ for 27 seconds. Two 128 formulations were manufactured, the standard formulation (PPSONS) and a formulation 129 without mineral $\operatorname{mix}(\mathrm{PPNONS})$. The total solids content of all products measured by 
130 refractometer, was $32 \%$. The $\mathrm{pH}$ ranged from 6.6 to 6.8 and density ranged from 1.05 and

$131 \quad 1.09 \mathrm{~g} / \mathrm{ml}$. All samples were stored at $4^{\circ} \mathrm{C}$ prior to tasting.

\section{$132 \quad$ 2.2. Sensory methods}

133 All sensory evaluation (sensory panel, healthy older volunteer and patient groups) was 134 carried out at room temperature $\left(25^{\circ} \mathrm{C}+/-2^{\circ} \mathrm{C}\right)$, product temperature was allowed to 135 equilibrate to room temperature; actual product temperature at serving was $20{ }^{\circ} \mathrm{C}(+/-3$ $\left.136{ }^{\circ} \mathrm{C}\right)$.

\section{$137 \quad$ 2.2.1 Sensory, volunteer and patient groups}

138 This study employed three different groups to assess the products; a trained sensory 139 panel, a healthy older volunteer panel and a patient group. The trained sensory panel 140 comprised 12 adults (11 females, 1 male; median age 42 years, range 33-59), expert in 141 profiling techniques, all had over 1 years experience and had been given a minimum of 4 142 hours training on profiling of ONS. The healthy older volunteer panel comprised 32 143 healthy, older, free-living volunteers (20 females, 12 males; median age 73 years, range $14466-88$ ). The patients were 28 older adults (11 female, 17 male; median age 85, range 71$14590)$ in hospital with a variety of medical conditions. Permission for the studies with the first 146 two panels was granted by the University of Reading Research Ethics committee and the 147 study with patients was approved by the Berkshire National Research Ethics committee 148 (NRES 08H0505176). All participants gave written informed consent prior to taking part in 149 the study.

150 Quantification with the trained panel took place in isolated booths, under artificial daylight 151 unless specified otherwise. Healthy older volunteer panels took place in a central location, 152 using isolated tables; lighting was standard fluorescent lighting. Patients were studied 153 individually at their bedside, under standard hospital lighting conditions.. 
155 The trained sensory panel characterised five specific sensory attributes of various ONS in

156 a sequential profile. This is a descriptive profiling method developed to determine the 157 perception of sensory attributes upon repeat consumption of ONS over time. Panellists 158 tasted eight consecutive aliquots $(5 \mathrm{ml})$ of each ONS sample and were instructed to score 159 the selected five attributes following each of the eight tastings. For each tasting, panellists 160 were also instructed to score the same five attributes as after-effects, following $30 \mathrm{~s}$ and $16160 \mathrm{~s}$ time delays. A two minute time delay was enforced between samples. Panellists 162 scored each attribute on unstructured line scales with the appropriate anchors. 163 Compusense five was used to design and run the profile and capture data.

164 The five attributes scored were sweet, metallic, soya milk flavour, mouthcoating and 165 mouthdrying. In a previous full quantitative descriptive analysis (QDA) profile of four 166 commercial products (Ensure Vanilla Plus, Abbott Nutrition UK; Fortisip Vanilla, Nutricia 167 Clinical Care UK; Resource Shake Vanilla and Clinutren Vanilla, Nestle Nutrition France) 168 sweet taste was found to be significantly different between samples $(p=0.03)$, soya milk 169 flavour was only found to be significant as an aftertaste $(p=0.03)$ (data not shown). QDA 170 did not reveal significant differences in metallic taste, mouthdrying or mouthcoating; and 171 yet these characteristics were thought to be distinct in ONS. The trained panel commented 172 on this and noted that these attributes appeared to last in the mouth beyond the profiling 173 session. It was, therefore, decided to study metallic, mouthdrying and mouthcoating, 174 alongside sweet taste and soya milk flavour, using the sequential profile.

175 Sequential profile data was collected for the following ONS: standard commercial vanilla 176 ONS (CONS) (Ensure Plus), sweetness suppressed vanilla ONS (SSONS; Lactisole in 177 Ensure; $0.003 \mathrm{~g} / 100 \mathrm{ml}$ ), pilot ONS control (PPSONS, with vitamins and minerals) and pilot 178 ONS with no mineral addition (PPNONS).

179 The commercial products (with and without lactose) were tasted in one week, in replicate, 180 samples presented in a balanced order. The pilot plant products were presented in 
181 replicate in a separate week, in balanced order. Samples were coded with 3-digit numbers;

182 however, all samples which were the same received the same code (panellist not blinded

183 to sequential protocol). Still mineral water and bread were provided as palate cleansers in-

184 between product samples (not between the eight consecutive aliquots of the same 185 sample). Panellists were instructed to drink all the sample volume presented and were not 186 permitted to drink water during sequential profiling.

$187 \quad$ 2.2.2.1 Sequential profiling method validation

188 In order to validate the sequential profiling method a further evaluation of CONS was 189 carried out, where panellists were given eight consecutive aliquots $(5 \mathrm{ml})$ of the same 190 sample, however, they were blinded to the test procedure, each aliquot had a unique three 191 digit code and these were presented in a balanced order. Time of tastings and scoring 192 after-effects were controlled in the same manner as the sequential profile.

\section{$193 \quad$ 2.2.3. Taste detection threshold tests}

194 For all groups, taste thresholds were determined by forced-choice ascending 195 concentration method (ASTM, 1997). Each assessor received a series of 3-alternative 196 forced choice (3-AFC) sets, each set comprised a taste solution (prepared in mineral 197 water) and two water samples at room temperature (balanced presentation order). Sets 198 were presented once, in order of increasing concentration, increased by a geometric 199 progression of two. Five iron sulfate (metallic) solutions were prepared from 2.8 to $20044.8 \mathrm{mg} / \mathrm{L}$, six sucrose solutions were prepared from 0.34 to $10.88 \mathrm{~g} / \mathrm{L}$ and ranges were 201 within those recommended by ISO 3972 (ISO, 1991). Patients received only sweet 202 solutions and only in five different concentrations, from 0.68 to $10.88 \mathrm{~g} / \mathrm{L}$. Samples were 203 coded with 3-digit random numbers. For metallic solutions, red light conditions were used 204 for the trained panel and sample cups with sip lids were used for the older volunteer panel. 205 Volunteers were instructed to choose the odd-one-out and comment on the taste which 206 they perceived in the most different sample. Individual detection thresholds were 
207 calculated as the geometric mean of the detection threshold and the concentration 208 preceding this

\subsubsection{Hedonic tests}

210 Hedonic liking data was collected from 32 healthy older adults and 28 patients, using a 211 modified boredom test (Köster and Mojet, 2007). This was used to characterise any 212 changes in liking of ONS following repeat consumption and to compare the liking of pairs 213 of samples. All subjects began by tasting $5 \mathrm{ml}$ of each of two samples (random 3 digit 214 coded, balanced presentation order) and scored liking for each on a 9-point hedonic scale 215 (initial liking), scaled from dislike extremely to like extremely. They then tasted a series of 216 eight consecutive $5 \mathrm{ml}$ aliquots of one sample (balanced presentation across volunteers, 217 samples coded by symbol) and were permitted to drink mineral water, if desired after 218 tasting the first four aliquots of the series of eight. Subjects subsequently tasted a further $2195 \mathrm{ml}$ of each of the two samples (random 3 digit coded, balanced presentation order), re220 scored their liking for each on the 9-point hedonic scale (final liking) and were asked to 221 state the sweetest sample of the final two samples. Subjects consumed $60 \mathrm{ml}$ of ONS in 222 total. The boredom trial was modified for the patient group in that the central eight $5 \mathrm{ml}$ 223 aliquots were replaced by a central cup containing the full $40 \mathrm{ml}$ of sample as it was 224 impractical to present 12 small cups on one tray at a patient's bedside. Patients were also 225 their sugar usage in tea and/or coffee.

\subsection{Statistical analysis}

227 SENPAQ (version 3.2) was used to carry out analysis of variance (ANOVA) and principal 228 component analysis (PCA) of sensory panel profiling data. In order to determine the 229 effects of time from the sequential profiling, three-way ANOVA was carried out in XLSTAT 230 (version 2009.1.02), using sample $(n=2)$, assessors $(n=12)$ and time $(n=8)$ as explanatory 231 variables. Non-parametric testing on the liking data and ANOVA on taste threshold data 232 were also carried out in XLSTAT (version 2009.1.02). 


\subsection{Sensory data}

\subsubsection{Sequential Profile}

\subsubsection{Standard ONS and sweetness suppressed ONS}

237 Sequential profile data was collected for commercial ONS (CONS) to characterise if 238 changes in perception of sensory attributes occurred over repeat consumption of a typical 239 commercial ONS. Sequential profiling was also carried out on the sweetness suppressed 240 variant (SSONS) to determine the effect of sweetness suppression on the perception of 241 sensory attributes over repeat consumption; the interest in sweetness suppression was 242 triggered by previous research which identified a disliking for the sweet taste of ONS 243 (Gosney, 2003; Methven et al, 2008).

244 Figure 1 illustrates how perception of the five selected sensory attributes varied with 245 repeat consumption of standard vanilla Ensure ONS (CONS). Mouthdrying, metallic, 246 mouthcoating and soya milk flavour built up significantly over time $(p<0.0001, p=0.002$, $247 p<0.0001$ and $p<0.0001$ respectively). Unlike the aforementioned attributes, sweetness did 248 not build over repeat consumption, it peaked at sips and decreasing as after-effects. 249 Figure 2 compares the standard sweet (CONS) and the sweetness suppressed (SSONS) 250 variants for three attributes. The SSONS was perceived as significantly less sweet 251 ( $p<0.0001$; initial mean scores 24 and 48 respectively). It was also significantly more 252 mouthdrying $(p<0.0001)$, although the difference was less substantial as tastings 253 progressed, (mean scores at second sip of 42 and 36 respectively). It is likely that the 254 sweeter sample is perceived as less mouthdrying due to the sweet taste interfering with 255 the drying perception; a previous study found sweetened soymilk to be less astringent than 256 its unsweetened counterpart (Courrelongue, Schlich and Noble, 1999). There was no 257 significant difference in the metallic perception of the two products, the soya milk flavour or 258 mouthcoating (data not shown). 


\subsubsection{ONS control and No-Mineral ONS formulations}

260 It was hypothesised that the minerals added to ONS during manufacture may contribute to 261 both astringent and metallic tastes. The mineral supplementation added to ONS contains 262 iron sulfate, known to impart metallic taste (Lim and Lawless, 2006). Minerals, particularly 263 zinc, are also known to impart astringent properties to solutions (Yang and Lawless, 2005). 264 To test the hypothesis, a control ONS formulation (PPSONS) that contained the full 265 mineral supplement and a formulation that had no mineral supplementation (PPNONS) 266 were manufactured. Figure 3 demonstrates the mouthdrying and metallic profiles of these 267 two ONS products. As with commercial ONS; metallic and mouthdrying built up 268 significantly over consumption time $(\mathrm{p}=0.001$ and $\mathrm{p}<0.0001$ respectively) for both products. 269 On first consumption $(5 \mathrm{ml})$ the mineral free product had a lower mean for metallic taste 270 (21.5 compared to 24.2) although the difference was not significant. Over all of the 271 consumption period (eight $5 \mathrm{ml}$ samples) the mineral free product (PPNONS) was 272 significantly less metallic $(p<0.0001)$, although the difference in overall means across time 273 was very small (25.2 and 26.7 respectively). It is therefore noted that although the minerals 274 added to the ONS formulation do contribute to the metallic taste, expected as the 275 supplementation contains iron sulfate, this cannot be the only source of metallic taste in 276 the products. The mineral supplementation is not thought to be the major source of 277 mouthdrying as the two products did not differ significantly in mouthdrying. It is 278 hypothesised that another source of mouthdrying could be the milk proteins typically used 279 in ONS formulations. Previous studies have shown whey proteins to cause mouthdrying 280 through precipitation onto the tongue (Sano, Egashira, Kinekawa and Kitabatake, 2005); 281 alternatively proteolysis of on $\beta$-casein can yield $\gamma$-caseins which are associated with 282 perceived dryness of milks (Harwalkar, Cholette, McKellar and Emmons, 1993). 
Given that panellists were asked to score the same attributes over time during sequential 285 profiling, their expectation might be that certain attributes were expected to build up over 286 time. However, in the first and subsequent sequential profile sessions four attributes 287 (mouthdrying, metallic, mouthcoating, and soya milk flavour) were found to build with time, whereas sweetness did not. It was not thought likely that the panellists anticipated that certain attributes would build over time and others would not. To further validate the 290 sequential profiling method, panellists were given eight consecutive aliquots of the same 291 sample, and blinded to the fact that the samples were identical. Figure 4 demonstrates that 292 the two methods did not give identical results. The panellists contributing to the data 293 acquired by both methods were the same, however, the batch codes of the samples were 294 different and the methods were run in different weeks. As the panellists were not using any 295 reference standard, it is expected that absolute values for the samples varied between the 296 methods; it is whether the trends differ that is important.

297 The two profiles (where panelists blinded to the sequential nature of the profile, and where 298 they were not blinded) gave very similar trends for sweetness; there was a significant 299 difference between results from the two methods $(p=0.001)$ and no significant change over 300 consumption time. For metallic taste, the panellists record a more substantial increase in 301 metallic taste over consumption time when not blinded to the sequential profiling, however 302 the trends for both methods was the same. There was a significant difference between the 303 two methods $(\mathrm{p}<0.0001)$, but still a significant overall increase in metallic taste with time $304(p<0.0001)$, with the not-blinded sequential profile finding a mean increase of 19 (from 18 305 to 37) and the blinded sequential profile a mean increase of 9 (from 13 to 21). Similarly for 306 mouthdrying and soy milk flavour (data not shown), there were significant differences in 307 the results from the two methods $(p=0.01, p=0.05)$, but a significant increase with 308 increased consumption overall $(p=0.026, p<0.0001)$. There was no significant difference 309 between the methods for mouthcoating (data not shown) and an overall increase in 
310 mouthcoating with increasing consumption $(p=0.001)$. In conclusion, it was found that

311 panellists may exaggerate increase in perception were they aware that they had

312 performed a sequential profile; however, the significant changes found over time were the

313 same whether panelists were blinded to the sequential nature or not.

314 Panellists received two ONS samples in one sequential profiling session; they therefore

315 consumed $80 \mathrm{ml}$ per session. This amount is in line with typical volumes of ONS consumed

316 in hospitals, as previously reported; Gosney (2003) identified that only $37 \%$ of ONS were

317 consumed, which is approximately $80 \mathrm{ml}$, assuming a typical pack size of $220 \mathrm{ml}$. The data

318 from the commercial sequential profiles is therefore likely to represent the sensory

319 characteristics perceived by patients consuming similar volumes of these products.

$320 \quad$ 3.1.2 Taste threshold tests

$321 \quad$ 3.1.2.1 Metallic taste thresholds

322 Metallic detection threshold tests were conducted to identify whether older consumers 323 could potentially identify the metallic attribute in the ONS, and to determine any difference 324 in metallic threshold between younger and older adults (Figure 5). However, it was 325 surprising that only $60 \%$ of the trained sensory panellists (median age 42) correctly 326 identified any sample differences in the metallic threshold test; the group best estimated 327 metallic threshold (geometric mean) for these panellists was $16 \mathrm{mg} / \mathrm{L}$. Forty percent of the 328 sensory panellists could not detect metallic at the maximum concentration of $45 \mathrm{mg} / \mathrm{L}$. In 329 comparison, only $32 \%$ of the healthy older volunteers (median age 73 ) correctly identified 330 any sample differences in the metallic threshold test; the group best estimated metallic 331 threshold for these volunteers was $26 \mathrm{mg} / \mathrm{L}$. The higher metallic detection threshold and 332 higher proportion of non-detectors observed in the group of healthy older volunteers was 333 expected as several studies have found elevated thresholds for taste and a diminished 334 ability to discriminate between suprathreshold stimuli (Schiffman and Graham, 2000). 335 However, the present study also questions the validity of using the 3-AFC test as a 
suitable test for metallic taste threshold determination. Metallic taste tends to be noticed as an aftertaste and, as shown in the sequential profiling results, it builds with time and is difficult to clear from the palate. Therefore, false identification is likely to arise from the 3AFC tests as a result of build up from previous samples tasted. If the sensory panellists were truly unable to detect iron sulfate as metallic at $45 \mathrm{mg} / \mathrm{L}$, it is unlikely that they would detect metallic taste in the ONS where the iron levels are typically around $20 \mathrm{mg} / \mathrm{L}$, unless most of the metallic taste perceived is not attributed to the iron sulfate. In a previous study ( $\mathrm{n}=18$, mean age 24$)$ the group best estimated threshold for iron sulfate was $27.5 \mathrm{mg} / \mathrm{L}$ (99 $\mathrm{mmol} / \mathrm{L})$, with a large standard deviation of $125 \mathrm{mg} / \mathrm{L}(452 \mathrm{mmol} / \mathrm{L})$ (Lim and Lawless, 2006); this study also used the 3-AFC test method.

\subsubsection{Sweet taste thresholds}

The mean sweet detection thresholds for the sensory panel (median age 42), healthy older volunteers (median age 73) and patients (median age 85 ) were $2 \mathrm{~g} / \mathrm{L}, 3 \mathrm{~g} / \mathrm{L}$ and $5.5 \mathrm{~g} / \mathrm{L}$ respectively. The median age of the older volunteers and patients combined was 78 years. The distribution of sweetness thresholds is given in Figure 6, which suggests an increase in sweet taste threshold with increasing age, as supported by previous literature (Zandstra and de Graaf, 1998; Mojet et al, 2003; Fukunaga et al 2005). Indeed, when the healthy older volunteers were divided into two age categories; 66 to 77 and 78 to 88 (below and above overall median age), the sweet taste thresholds were 2.6 and $4.1 \mathrm{~g} / \mathrm{L}$ respectively, although this difference was not significant. The higher taste thresholds of the patients compared to the older volunteers cannot be explained by age alone. Combining the healthy older volunteer and patient data together and analysing for the effect of group (healthy or patient) and age (< or $>78$ ) by ANOVA; the group had a significant effect on sweet taste threshold $(p=0.005)$, whereas the age did not. It is, therefore, hypothesised that illness and medication have a greater effect on sweetness thresholds than age. IIIness and medication are known to taste thresholds increases as well as a wide range of taste 
disturbances; this area has been previously reviewed by Schiffman (Schiffman and Zervakis, 2002). The patient cohort were prescribed an average of 4.5 medications (range $0-11$ ) of which an average of 1.4 (range 0 to 3 ) were known have the capacity to cause taste disturbance (British National Formulary, 2009). The healthy older volunteers were prescribed an average of 2.1 medications (range $0-11$ ) of which an average of 0.7 (range 0 to 3) had the capacity to cause taste disturbance.

\subsection{Hedonic data}

\subsubsection{Boredom test}

Mean liking scores for standard ONS (CONS) and the sweetness suppressed ONS (SSONS), at start and end of the boredom test, are given in Table 3. With both older cohorts, the mean initial liking of the standard vanilla ONS was significantly higher than the initial liking of the sweetness suppressed ONS ( $p \leq 0.05)$. However, there was a difference between the cohorts in their change in liking from start to end of the boredom test. The healthy volunteer mean liking of the standard ONS significantly decreased during the boredom test from 6.3 to 5.0 ( $p \leq 0.001$ ). This was irrespective of whether they received 40 $\mathrm{ml}$ of CONS or SSONS during the boredom test (sample received in-between the initial and final liking pairs). The liking of the SSONS did not change over time for the volunteer cohort. In contrast, there was not a decrease in liking of the standard product during the boredom test for the patient cohort. However, their liking of the SSONS did decrease significantly over the boredom test, irrespective of the boredom sample $(p \leq 0.05)$. One point to note in carrying out the boredom trials with the patient group, as the central boredom sample was contained in one cup as a $40 \mathrm{ml}$ sample, rather than as eight individual $5 \mathrm{ml}$ samples, there was a tendency for patients not to consumer the full $40 \mathrm{ml}$ which is likely to have reduced any effect of change in liking over the boredom test.

The main conclusions from the boredom liking tests were that overall liking of the CONS was greater than the SSONS. As liking was found to decrease with repeat consumption, it 
388 is likely that consumption of a typical pack volume may reduce liking of the products even

389 further. It is hypothesised that the attributes of mouthdrying and metallic which were found 390 in the sequential profiling study to build substantially over consumption volume may, in 391 part, cause the reduction in liking.

\subsubsection{Consideration of sugar usage and sweetness thresholds on ONS liking}

393 There were 17 patients who regularly took sugar in their tea or coffee and 11 who did not.

394 There was no correlation between sugar usage and sweetness threshold. In addition, 395 there was no correlation between sugar usage or sweetness threshold and liking scores 396 for the standard ONS in comparison to the sweetness suppressed variant. The later point 397 supports the previous study by Mojet et al (2005) which found sweetness thresholds not to 398 correlate with preferred sweetness level. The volunteers and patients could determine that 399 the standard ONS sample was sweeter than the SSONS $(p<0.0001 ; 26$ out of 32 400 volunteers; 25 out of 27 patients). Healthy older volunteers and patients who incorrectly 401 identified which sample was the sweetest, did not have the highest sweetness thresholds; 402 implying that the sweetness of the products was above each individuals sweetness 403 threshold. This also demonstrates that sugar consumption in hot beverages did not impact 404 upon ONS sweetness perception.

\section{4. Conclusions}

406 Sequential profiling was used to characterise five attributes of vanilla dairy-based ONS 407 over repeat consumption. This highlighted a significant build up of mouthdrying, metallic 408 and mouthcoating attributes over a total consumption volume of $40 \mathrm{ml}$, which would not 409 have been found though a standard profiling study. Such build may have major 410 implications on the long-term, repeat consumption of these products, especially since 411 patients are often encouraged to drink up to $600 \mathrm{ml}$ daily. Liking of ONS, with both healthy 412 older and older patient groups, was found to diminish over repeat consumption (60ml), 413 suggesting that build up of taste and mouthfeel attributes over repeat consumption was 
414 disliked. The combined use of sequential profiling and liking over repeat consumption 415 (using a boredom test approach) is recommended as a methodology suitable for the exploration of products such as ONS which are known to have aftertastes.

417 Removal of the minerals from an ONS formulation did not significantly reduce mouthdrying 418 and although the effect on metallic taste perception was significant, it was not substantial. 419 Components other than iron sulfate, intrinsic to ONS, such as the calcium and milk 420 proteins, may contribute to these attributes. In support of this, calcium salts have been 421 shown to exhibit both astringent and metallic taste properties (Lawless, Rapacki, Horne 422 and Hayes, 2003) and both whey protein precipitation and casein proteolysis products 423 have been associated with mouthdrying (Sano et al, 2005; Harwalkar et al, 1993). Further 424 research into the properties of ONS ingredients may help to elucidate potential causes of 425 the build up of attributes over repeat consumption. If the build up can be reduced this may 426 lead to improve palatability and consumption of ONS.

427 Acknowledgments: This work has been supported by Research into Ageing (Age UK), a 428 UK Charity. Abbott Nutrition and Nestle Nutrition are thanked for supply of ingredients, 429 samples and advice. Thank you to Dr Chris Findlay and the Compusense support team for 430 their assistance in setting up the sequential profile.

\section{$431 \quad$ References}

Age Concern (2006). Hungry to be Heard; the scandal of malnourished older people in hospital. Age Concern, London.

ASTM Committee (1997) Standard practice E 679-91. Determination of odor and taste thresholds by a forced-choice ascending concentration series methods of limits. Philadelphia, PA: American Society for Testing and Materials.

Bolton, J., Abbott, J.R., Kiely. M., Alleyne,M. Bel,S., Stubbs,L. \& Slevin, M. (1992) Comparison of three oral sip-feed supplements in patients with cancer. Journal of Human Nutrition and Dietetics, 5 (2)79-84.

British National Formulary (2009) BNF 58, London : BMJ Group and RPS Publishing.

Courrelongue, S., Schlich, P. \& Noble, A.C. (1999) Using repeated ingestion to determine the effects of sweetness, viscosity and oiliness on temporal perception of soymilk astringency. Food Quality and Preference, 10, 273-279.

Duizer, L., Bloom, K. \& Findlay, C.J. (1991) Dual-attribute time-intensity sensory evaluation: A new method for temporal measurement of sensory perceptions. Food Quality and Preference, 8(4),261-269. 
Fearon, K.C., Meyenfeldt, M.F., Moses, A.G., Van Geenen, R., Roy, A., Gouma, D.J., Giacosam, A., Van Gossum, A., Bauer, J., Barber, M.D., Aaronson, N.K., Voss, A.C. \& Tisdale, M.J. (2003) Effect of a protein and energy dense $\mathrm{N}-3$ fatty acid enriched oral supplement on loss of weight and lean tissue in cancer cachexia: a randomised double blind trial. Gut, 52, 1479-1486.

Fukunaga, A., Uematsu, H \& Sugimoto, K. (2005) Influences of aging on taste perception and oral somatic sensation. Journal of Gerantology, 60A(1), 109-113.

Gosney, M. (2003) Are we wasting our money on food supplements in elder care wards? Journal of Advanced Nursing, 43(3), 275-280.

Harwalkar, V. R., Cholette, H., McKellar, R.C. \& Emmons, D.B. (1993) Relation Between Proteolysis and Astringent Off-Flavor in Milk. J. Dairy Sci. 76: 2521-2527.

International Standard ISO 3972 (1991) Methods for sensory analysis of food - Part 7: Investigating sensitivity of taste.

Jack, F.R., Piggott, J.R. \& Paterson, A. (1994) Analysis of textural changes in hard cheese during mastication by progressive profiling. Journal of Food Science, 59(3), 539-543.

Kaneda, H., Maeshima, K., Goto, N., Kobayakowa, T., Ayabe-Kanamura, S. \& Saito, S. (2000) Decline in taste and odour discrimination abilities with age, and relationship between gestation and olfaction. Chemical Senses, 25, 331-337.

Köster, E.P. \& Mojet, J. (2007) Boredom and the reasons why some new food products fail. In: Consumerled food product development (p262-280). Cambridge: Woodhead Publishing Limited.

Koskinen, S., Kälviänen, N. \& Tuorila, H. (2003) Flavour enhancement as a tool for increasing pleasantness and intake of a snack product amongst the elderly. Appetite, 41, 87-96.

Labbe, D., Schlich, P., Pineau, N., Gilbert, F.\& Martin, N. (2009) Temporal dominance of sensations and sensory profiling: A comparative study. Food Quality and Preference, 20, 216-221.

Law, C.K-W., Gosney, M.A. \& Kennedy, O.B. (2006) Age related changes in taste and effect on food supplement palatability. Proceedings of the Nutrition Society, 65, OCB, 56A.

Law, C.K-W., (2006) Age related changes in taste and effect on food supplement palatability. Dissertation for BSc Degree in Food Science, University of Reading.

Lawless, H., Rapacki, F., Horne, J. \& Hayes, A. (2003) The taste of calcium and magnesium salts and anionic modifications. Food Quality and Preference, 14(4), 319-325.

Lim, J. \& Lawless, H. (2006) Detection thresholds and taste qualities of iron salts. Food Quality and Preference, 17,513-521.

Lochs, H., Alliaon, S.P., Meier ,R., Pirlich, M., Kondrup, J., Schneider, St., Berghe, G., \& Pichard, C. (2006) Introductory to the ESPEN Guidelines on Enteral Nutrition: Terminology, Definitions and General Topics. Clinical Nutrition, 25(2), 180-186.

Methven, L., Kennedy, O.B., Mottram, D.S., Grimble, G.K., Bushell, M., Gray, L. \& Gosney, M.A. (2008) The effect of saccharide type on sweet perception and preference of Oral Nutritional Supplements by elderly people. Proceedings of the Nutrition Society, 68 (OCE1) E55.

Milner, A.C., Potter, J., Vivanti, A., \& Avenell,A. (2002) Protein and energy supplementation in elderly people at risk of malnutrition. Cochrane Database Systematic Reviews 2007, Issue 4. Art. No.:CD003288. DOI: 10.1002/14651858. CD003288.pub3.

Mojet, J., Heidema, J. \& Christ-Hazelhof, E. (2003) Taste perception with age: generic or specific losses in supra-threshold intensities of five taste qualities ? Chemical Senses. 28, 397-413. 
Mojet, J., Christ-Hazelhof, E , \& Heidema, J. (2005) Taste perception with age: pleasantness and its relationships with threshold sensitivity and supra-threshold intensity of five taste qualities. Food Quality and Preference, 16, 413-423.

Nolan, A. (1999). Audit of supplement use on care of the elderly and rehabilitation wards. Journal of Human Nutrition and Dietetics, 12(5), 453-458.

O'Flynn, J., Peake, H., Hickson, M., Foster, D., \& Frost, G. (2005) The prevalence of malnutrition in hospitals can be reduced: Results from three consecutive cross-sectional studies. Clinical Nutrition, 24(6), 1078-1088.

Rahemtulla, Z., Baldwin, C., Spiro, A., McGough, C., Norman, A.R., Frost, G., Cinningham, D., \& Andreyev, H. (2005) The palatability of milk-base and non-mil-based nutritional supplements in gastrointestinal cancer and the effect of chemotherapy. Clinical Nutrition, 24, 1029-1037.

Sano, H., Egashira, T., Kinekawa Y. \& Kitabatake, N. (2005) Astringency of Bovine Milk Whey Protein. $\underline{J}$. Dairy Sci. 88: 2312-2317.

Schiffman, S.S. \& Graham, B.G. (2000) Taste and smell perception affect appetite and immunity in the elderly. European Journal of Clinical Nutrition, 54(3), S54-S63.

Schiffman, S.S. \& Zervakis (2002) Taste and smell perception in the elderly: effect of medication and disease. Advances in Food and Nutrition Research, 44, 247-346.

Stableforth, P.G. (1986). Supplement feeds and nitrogen and calorie balance following femoral neck fracture. British Journal of Surgery, 72, 651-655.

Yang, H.H-L, \& Lawless, H. (2005) Descriptive analysis of divalent salts. Journal of Sensory Studies, 20, 97113.

Zandstra, E.H. \& de Graaf, C. (1998) Sensory perception and pleasantness of orange beverages from childhood to old age. Food Quality and Preference, 9(1/2) 5-12.

\section{Figure 1: Sequential profile of commercial vanilla ONS}

\section{Footnote figure 1:}

${ }^{(a)}$ Sip 1-8, consumption point of $5 \mathrm{ml}$ aliquots; AE1, AE2, after-effects at $30 \mathrm{~s}$ and $60 \mathrm{~s}$ post consumption of aliquots 1-8.

\section{Figure 2: Sequential profiles of two sweetness variants of vanilla ONS}

\section{Footnote figure 2:}

(a) Sip 1-8, consumption point of $5 \mathrm{ml}$ aliquots; $A E 1, A E 2$, after-effects at $30 \mathrm{~s}$ and $60 \mathrm{~s}$ post consumption of aliquots 1-8. CONS = Standard commercial ONS ; SSONS = Sweetness Suppressed commercial ONS

\section{Figure 3: Sequential profiles of two mineral variants of vanilla ONS}

\section{Footnote figure 3:}

(a) Sip 1-8, consumption point of $5 \mathrm{ml}$ aliquots; AE1, AE2, after-effects at $30 \mathrm{~s}$ and $60 \mathrm{~s}$ post consumption of aliquots 1-8. PPSONS = control pilot plant ONS; PPNONS $=$ No mineral pilot plant ONS 
527 Figure 4: Validation of sequential profiling, used to quantify three attributes of

528 commercial vanilla ONS over eight consecutive $(5 \mathrm{ml})$ consumptions

529 Footnote figure 4:

$530 \quad{ }^{(a)}$ Sip 1-8, consumption point of $5 \mathrm{ml}$ aliquots; AE1, AE2, after-effects at $30 \mathrm{~s}$ and $60 \mathrm{~s}$ post consumption 531 of aliquots $1-8$. Blind= panellists not aware that consecutive samples were the same sample; Sequential $=$ panelists aware profile was sequential

535 panellists and healthy older volunteers

537 Figure 6: Frequency distribution of sweet taste detection thresholds for sensory

538 panellists, healthy older volunteers and patients 
Table 1. Liking Scores for Standard Commercial (CONS) and Sweetness Suppressed (SSONS) ONS at Initial and End Tasting, following a Boredom Test, for a healthy older volunteer cohort $(n=32)$ and a patient cohort $(n=28)$

\begin{tabular}{|c|c|c|c|c|c|c|c|c|c|c|}
\hline \multirow[b]{2}{*}{ Cohort } & \multirow[b]{2}{*}{ Product } & \multicolumn{3}{|c|}{$\begin{array}{l}\text { Mean Liking }{ }^{\text {a }} \text { (Irrespective of } \\
\text { sample used for } 40 \mathrm{ml} \\
\text { boredom) }\end{array}$} & \multicolumn{3}{|c|}{$\begin{array}{c}\text { Mean Liking }^{\mathrm{b}} \text { (Participants consuming } \\
\text { CONS } \\
\text { for Boredom phase) }\end{array}$} & \multicolumn{3}{|c|}{$\begin{array}{c}\text { Mean Liking }^{\mathrm{C}} \text { (Participants consuming } \\
\text { SSONS } \\
\text { for Boredom phase) }\end{array}$} \\
\hline & & Initial & Final & $\operatorname{Sig}^{\mathrm{d}}$ & Initial & Final & $\operatorname{Sig}^{\mathrm{d}}$ & Initial & Final & $\operatorname{Sig}^{d}$ \\
\hline Healthy & CONS & $6.3 \pm 1.7$ & $5.0 \pm 1.9$ & $* * *$ & $6.4 \pm 1.4$ & $5.6 \pm 1.4$ & * & $6.1 \pm 2.0$ & $4.3 \pm 2.2$ & ** \\
\hline Older & SSONS & $5.2 \pm 1.9$ & $5.5 \pm 1.9$ & ns & $5.2 \pm 1.7$ & $5.0 \pm 1.6$ & ns & $5.3 \pm 2.2$ & $6.1 \pm 2.1$ & ns \\
\hline Volunteers & $\mathrm{Sig}^{\mathrm{e}}$ & * & ns & & * & ns & & ns & $n s(p=0.08$ & \\
\hline \multirow[t]{3}{*}{ Patients } & CONS & $6.8 \pm 1.8$ & $6.7 \pm 1.8$ & ns & $6.9 \pm 1.3$ & $6.3 \pm 1.2$ & ns $(p=0.06)$ & $6.8 \pm 2.2$ & $7.0 \pm 2.1$ & ns \\
\hline & SSONS & $6.1 \pm 1.9$ & $5.2 \pm 2.3$ & * & $6.2 \pm 1.3$ & $4.9 \pm 2.0$ & * & $6.1 \pm 2.2$ & $5.4 \pm 2.5$ & ns \\
\hline & $\operatorname{Sig}^{\mathrm{e}}$ & * & $* * *$ & & ns & * & & ns $(p=0.06)$ & * & \\
\hline
\end{tabular}

${ }^{\text {a }}$ Preference data represents mean scores \pm standard deviation, from a 9-point hedonic scale for all volunteers; ${ }^{\mathrm{b}} 18$ volunteers and 12 patients consumed CONS

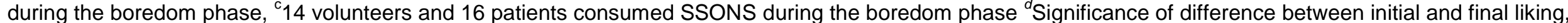
as shown by ANOVA: $\mathrm{p}<0.001\left(^{* *}\right), \mathrm{p}<0.01\left(^{* *}\right), \mathrm{P}<0.05\left({ }^{*}\right)$, not significant (ns). . ${ }^{e}$ Significance of difference between CONS and SSONS. 